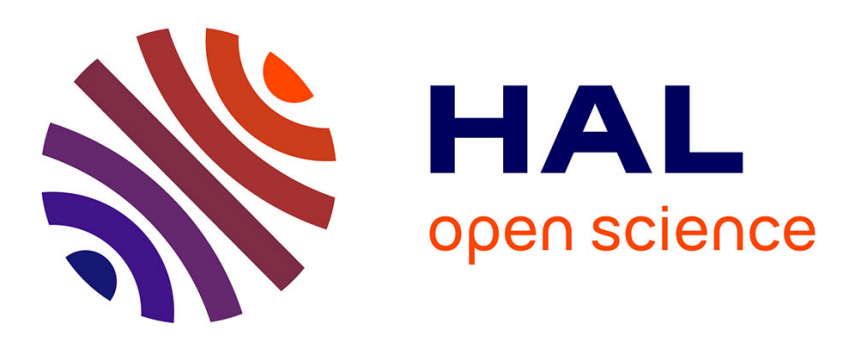

\title{
Towards a qualitative spatial model for road traffic in urban environment
}

\author{
Kamaldeep Singh Oberoi, Géraldine del Mondo, Yohan Dupuis, Pascal \\ Vasseur
}

\section{- To cite this version:}

Kamaldeep Singh Oberoi, Géraldine del Mondo, Yohan Dupuis, Pascal Vasseur. Towards a qualitative spatial model for road traffic in urban environment. Proceedings of 2017 IEEE 20th International Conference on Intelligent Transportation Systems (ITSC), IEEE, Oct 2017, Yokohama, Japan. pp.1724-1729, 10.1109/ITSC.2017.8317644 . hal-02119842

\section{HAL Id: hal-02119842 \\ https://hal.science/hal-02119842}

Submitted on 4 May 2019

HAL is a multi-disciplinary open access archive for the deposit and dissemination of scientific research documents, whether they are published or not. The documents may come from teaching and research institutions in France or abroad, or from public or private research centers.
L'archive ouverte pluridisciplinaire HAL, est destinée au dépôt et à la diffusion de documents scientifiques de niveau recherche, publiés ou non, émanant des établissements d'enseignement et de recherche français ou étrangers, des laboratoires publics ou privés. 


\title{
Towards a Qualitative Spatial Model for Road Traffic in Urban Environment
}

\author{
Kamaldeep Singh Oberoi*, Géraldine Del Mondo ${ }^{\dagger}$, Yohan Dupuis ${ }^{\ddagger}$ and Pascal Vasseur ${ }^{\S}$ \\ ${ }^{*}{ }^{\$}$ Laboratoire d'Informatique, de Traitement de l'Information et des Systèmes \\ ${ }^{*}$ Normandie Univ, UNIROUEN, UNIHAVRE, INSA Rouen, LITIS, 76000 Rouen, France \\ ${ }^{\dagger}$ Normandie Univ, INSA Rouen, UNIROUEN, UNIHAVRE, LITIS, 76000 Rouen, France \\ $\ddagger$ CEREMA, 76120 Le Grand-Quevilly, France
}

\begin{abstract}
This paper presents preliminary steps towards the development of a general spatial model, based on graph theory, to visualize and reason about the road traffic in an urban environment. This model includes qualitative, in addition to quantitative, data which improves its computation and makes it robust to quantitative errors. The paper also describes different levels of abstraction which define distinct points of view of the environment and hence, allow for the acquisition of heterogeneous data. The graph consists of set of entities and corresponding spatial relations, the semantics behind which differ according to the level of abstraction. The ideas presented in this paper bring together the research done by Geomatics and Robotics/Perception communities.
\end{abstract}

\section{INTRODUCTION}

The desire to understand and formalise the evolution of vehicular traffic flow as well as the environment around a single vehicle has always been there among various research communities. Physicists developed microscopic traffic flow models like cellular automaton (CA) model, also called NagelSchreckenberg (Na-Sch) model [1], to represent the motion of a single vehicle hopping from one cell to the next in one time step, over a freeway. [2] describes a model which considers the velocity distribution of multi-lane vehicular traffic, and forms a basis for other macroscopic traffic flow models. Researchers working with Geographical Information Systems (GIS) have also shown interest in including the continously varying traffic data within a geographical database [3], [4]. The results of DARPA Grand Challenge (2005) have motivated robotics community to contribute in the maturation of Intelligent Transportation Systems [5]. The development of comparatively cheaper and more accurate sensors along with better performing machine learning algorithms has made driver assistance systems more advanced and reliable [6].

For the development of autonomous vehicles, quantitative data acquired from the on-board and/or external sensors is used as it provides the exact measurements to "understand" the environment [7], [8]. While it provides precise knowledge, quantitative data is prone to errors and proves less useful if we want to reason in qualitative terms. [9] presents the idea of using both qualitative and quantitative information to model the characteristics of traffic flow. It describes traffic parameters which can only be defined at macroscopic level. Other models, like the one presented in [10], take into account only the microscopic data for the traffic flow.

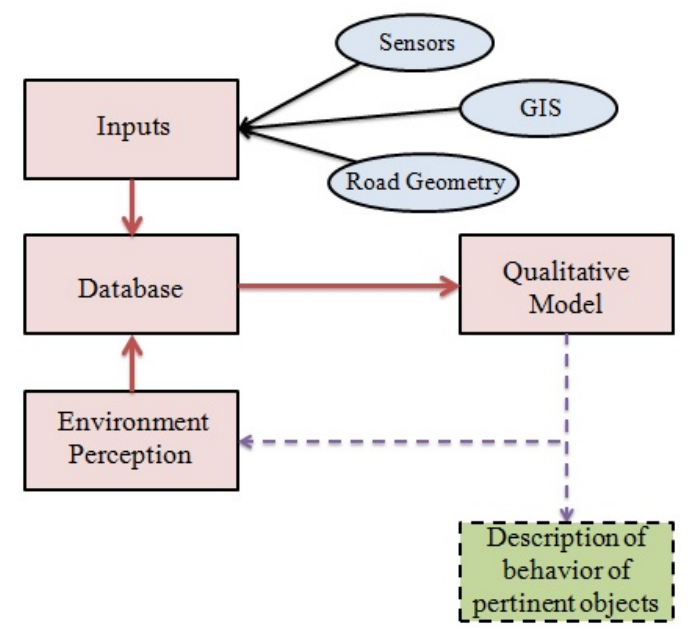

Fig. 1. General block diagram of a global system

The model introduced in this paper, however, considers qualitative as well as quantitative data about the road traffic at both microscopic and macroscopic levels. The ideas put forward in this paper, which also define the objectives for the ongoing work, tend towards combining the spatial information about the environment around a single vehicle (microscopic level) along with that associated to a road network located in a geographical area (macroscopic level) and the traffic data for that road network, in a single model, which will be useful to perceive the environment around a vehicle more robustly and to better understand the flow of traffic in that area. In addition, qualitative relations, defined for a range of quantitative values, will be useful to avoid fluctuation/errors (if any) in those values. In this regard, the information gathered by different GIS about the urban road network (like traffic density, travel time etc.), along with vehicle's internal (e.g. GPS, internal cameras etc.) and external (e.g. inductive loop counters, external cameras etc.) sensors, will be considered. The geometric design of road (like width, length, sight distance, number of lanes etc.) will also be included in the model.

Fig. 1 shows a general block diagram of a global system of which the qualitative model is a part. The inputs module specifies different sources of inputs from which the data will be collected and stored in the database. This database will also 
include the output of different perception algorithms, such as the distance between two vehicles, position of a vehicle with respect to another vehicle, relative trajectory of two vehicles etc. All this information will act as quantitative input for the qualitative model, which will be used to describe the behavior of pertinent objects (like vehicles) under different traffic conditions, and in-turn improve the environment perception. The data structure used to visualize the model and its constituents at different levels of abstraction is a graph, whose nodes represent the entities and edges the spatial relations. Graphs provide a good framework for qualitative reasoning as the relations between different traffic participants can be easily represented and the spatial changes between them can be efficiently computed.

This paper is organized as follows: In section II, preliminary ideas for the development of the qualitative model are described. Section III talks about two types of granularity which are present in the model. It also discusses different entities and spatial relations considered at different levels of abstraction. The paper is concluded and the future work is mentioned in section IV.

\section{Qualitative Model}

In this section, we will describe the qualitative spatial graph model. We will first take a look at the entities and spatial relations included in the model. Then we will discuss two ways in which a road network can be visualized graphically. In the end, the mathematical formalization of the graphs at different levels of abstraction will be defined.

\section{A. Entities}

Entity represents an object (physical/abstract) which plays an important role in understanding the phenomenon being modeled. In different domains, entities have different definitions. A geographic entity is an object which occupies some finite space [11], while in DBMS, it represents a thing of interest about which the data has to be stored in the database [12]. There can be multiple entities in a model which may have similar characteristics/properties, but they must be differentiated by defining a unique identity [13]. In our model, entity represents different things at different levels of detail, as described in later sections. A unique label provides the identity to each entity.

\section{B. Spatial Relations}

The entities interact with each other in space. These interactions form the spatial relations between pairs of entities and are represented as edges in the graph. There are different types of spatial relations which are included in the model. [14] defines three types of spatial relations: (1) metric relations, which take into account quantitative aspects of space (2) topological relations, which give qualitative information about geometrical interactions between two spatial objects and (3) order relations, which specify the relative position of two spatial objects.

Another type of relations which specify the relative position of two spatial entities is orientation relations [15], [16]. These

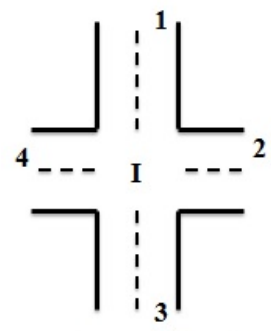

Road Network with an intersection

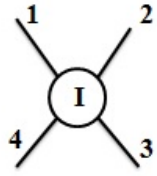

Primal Graph

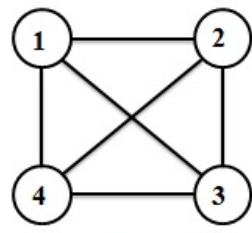

Dual Graph
Fig. 2. Primal and Dual graph representation for an arbitrary road network

differ from directional relations [17] as orientation relations use an explicit frame of reference present at the same location as the reference object, whereas directional relations use a general frame of reference. For example, an orientation relation leftof is defined according to the shape or geometry of an object but a directional relation northOf considers the well defined cardinal directions. [18] describes an approach to include qualitative distance between two entities and define relations such as close, far, very far etc.

When there is a change in the position of the entities over time, then a new kind of relation which defines the relative direction of motion between two entities comes into picture, and can be described using Directed Interval Algebra (DIA) [19]. DIA, using spatial intervals, gives another framework to represent relative position of extended objects.

The list of spatial relations described in this section is by no means exhaustive. There are other spatial relations which are not mentioned in this section, but are described later wherever they are used.

\section{Representation of urban road networks}

Now we will take a look at two approaches, defined in the literature, to represent the urban road networks using a graph model.

In the first approach, a road network is represented by a graph whose nodes are the intersections and the edges are the roads segments joining the two adjacent intersections. [20] calls this graph as primal graph. Primal graphs exhibit the actual spatial structure of the network and, due to their intuitiveness for human understanding, are widely used and, thus, form a basis for a large number of datasets. Parameters such as travel time, traffic density, distance etc. can be incorporated into the edges of the graph.

The second approach to represent a road network considers road segments as nodes and if two road segments intersect, an edge is added between the associated nodes. This graph is called dual graph [21]. Dual graphs represent the topological structure of the network and can be used to measure the 
centrality or importance of a road segment. Fig. 2 shows primal and dual graphs for an arbitrary road network consisting of four numbered road segments intersecting with each other.

In a primal graph, the information about the road segment like length, travel time, width, sight distance etc. will be associated with an edge of the graph which makes it more natural for human understanding, and the information about the type of intersection ( $T, X$, Roundabout etc.) can be defined as a property of a node. However, dual graph exhibits the information about the spatial relations between two road segments and helps in visualizing the traffic flow at an intersection. We consider both approaches in our model.

\section{Definitions of important terms}

Before describing the model, we would like to define some terms used in the model which are also represented in Fig. 3.

Road segment: A road segment is defined as a part of the road network which connects two intersections i.e., the two adjacent intersections are considered as the end points of the road segment connecting them. A road segment could be divided into two carriageways or it could be divided into sectors. Both these divisions give rise to two different points of view using which the granularity in the model is defined in later sections.

Road carriageway: When a road segment is divided into two carriageways, it represents the bidirectional nature of the traffic flow [22]. These carriageways may or may not be multilane. Let $L$ be a set of carriageways for a road segment, then we define: $L=\{l 1, l 2\}$, where $l 1$ and $l 2$ are opposite direction carriageways.

Road sector: A road segment can be divided into consecutive non-overlapping sectors (similar as in [23]). The division can be on the basis of the length of the sector, or geometric road design. Let $A$ be a set of sectors over a road segment, we have: $A=\left\{A_{1}, \ldots, A_{n}\right\}$, where $A_{i}$ is a sector. The number of sectors for a road segment varies with the level of abstraction.

\section{E. Spatial Graphical Model}

Having defined the concepts and definitions to be used in the model, in this section we will take a look at how these concepts are interwoven together.

A graph for a given urban area is defined as

$$
G=(X, E)
$$

where $X=V \cup B \cup V S \cup M \cup F \cup P \cup H \cup$ $R \cup I$ represents the set of nodes. Here $V$ is set of vehicles, $B$ is set of buildings, $V S$ is set of vertical structures (e.g. lane dividers, signboards, traffic signals, guard rails), $M$ is set of road markings (e.g. zebra crossing, edge line, stop line, center line), $F$ is set of roadsides (e.g. footpath, bicycle track, vegetation), $P$ is set of pedestrians, $H$ is set of bicycles, $R$ is set of road segments and $I$ is the set of intersections. The elements of $X$ pertain to different object classes present in a typical urban scenario. These object classes are chosen to be included in the model as they have a direct influence on traffic flow.

$$
E=\{(x, y) \mid x \rho y\}, \rho \in \varrho
$$
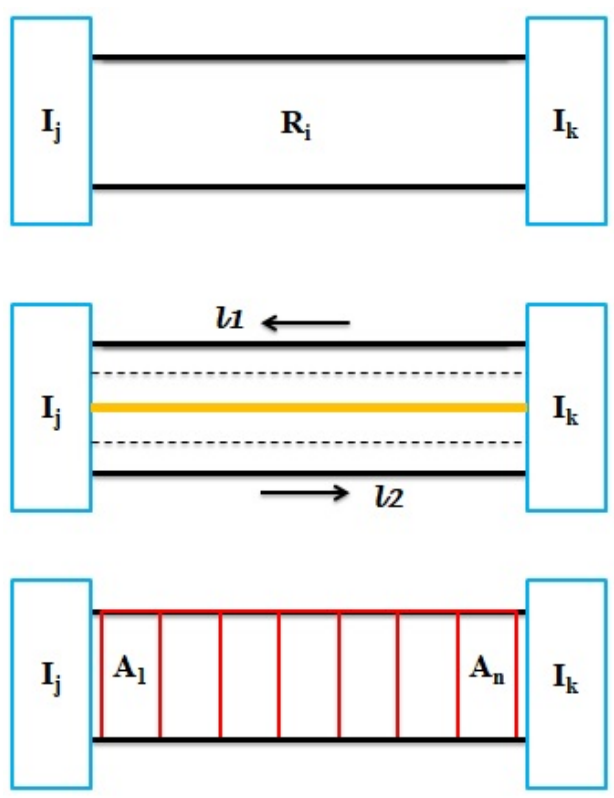

Fig. 3. Two ways to divide the road segment. A road segment $R_{i}$ between two intersections $I_{j}$ and $I_{k}$ (top) can be divided into two carriageways $l 1$ and $l 2$ with traffic flow in opposite directions (middle), or into nonoverlapping sectors (bottom). Here, the rectangular blocks used to represent the intersections are just symbolic.

$$
\forall x, y((x \in V \wedge y \in X) \vee(x \in F \wedge y \in P \cup H))
$$

represents the set of edges (relations) between the elements of $X$, and the set of all possible types of such relations is given by $\varrho=\{T, O, R T, R S, Q D, O r d\}$ where $T$ is set of topological relations (defined, for example, using RCC8) [24], $O$ is set of orientation relations, set $R T$ represents relative trajectories, set $R S$ describes the relative speed (slow, fast etc.) [25], set $Q D$ defines qualitative distance relations, and Ord defines set of order relations [26].

Considering a single road segment $R_{i} \in R$, a graph containing physical objects as entities and corresponding relations is defined as

$$
G_{i}=\left(X_{i}, E_{i}\right)
$$

where $X_{i} \subseteq X \backslash\left(I \cup\left\{R_{j} \mid R_{j} \in R, j \neq i\right\}\right), i, j=1, . ., N_{R}$ is the set of entities with $N_{R}$ being the total number of road segments in $R$ and $E_{i} \subseteq E$ is the set of edges. In $X_{i}$, all the roads except $R_{i}$ and all the intersections are ignored. It is noteworthy that the type of relations considered in both $G$ and $G_{i}$ are same.

If a road segment $R_{i} \in R$ is visualized in terms of carriageways $L=\{l 1, l 2\}$, then a corresponding two-carriageway graph

$$
G_{L i}=\left(Y_{i}, E_{\omega i}\right)
$$

has two groups of vehicles traveling in opposite directions as nodes, and the edge represents the relation between these groups. The set of nodes is $Y_{i}=\left\{V_{l 1}, V_{l 2}\right\}$ where $V_{l 1}$ 


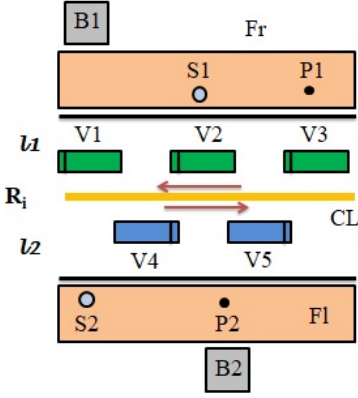

(a)

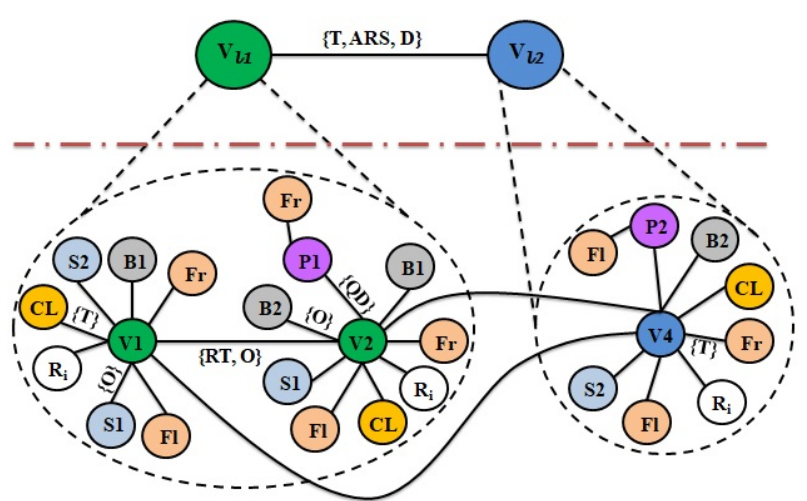

(b)

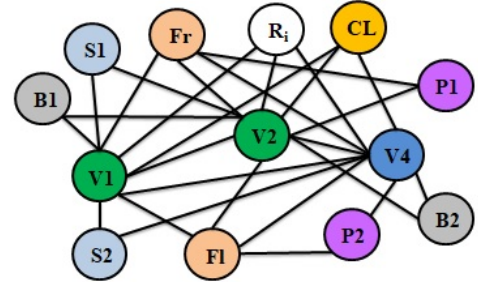

(c)

Fig. 4. (a) A road segment divided into two carriageways (b) A graph showing two levels of abstraction. At the finer level, a part of $G_{i}$ is shown for vehicles $V 1, V 2$ and $V 4$. At a coarser level, the vehicles get categorized into two groups depending on their relation with the carriageways. All the relations are not shown to make the image more readable (c) Storage and implementation view of the graph in (b)

and $V_{l 2}$ consider the graphs associated to all the vehicles in carriageway $l 1$ and $l 2$, respectively (Fig. $4 \mathrm{~b}$ ). The edge set is

$$
E_{\omega i}=\{(x, y) \mid x \omega y\}, \omega \in \Omega, \forall x, y \in Y_{i}, x \neq y
$$

and the set of types of relations between these groups is $\Omega=$ $\{T, A R S, D\}$. Here, set $A R S$ represents the average relative speed between two groups and $D$ is the set of directional relations.

When we divide a road segment into sectors, a graph for each sector is defined which contains the vehicles and physical objects included in that sector. The sector graph for $j$ th sector of $i$ th road segment $R_{i} \in R$ is defined as

$$
G_{A i j}=\left(U_{i j}, E_{i j}\right)
$$

Since $U_{i j} \subseteq X_{i}$ and $E_{i j} \subseteq E_{i}$, we can say that $G_{A i j} \subseteq G_{i}$. Depending on the level of abstraction, individual sectors can be combined/segregated to form new sectors each with their own associated graphs (described later).

Now, let's see the graph formalization for primal and dual graphs which are useful to understand the structure of the road network. It should be noted that both these graphs are not the subgraphs of $G$, but are used to enhance the knowledge which $G$ represents. Moreover, these are static graphs, whereas $G$ will be time varying (temporal information will be included in the model in future).

In primal graph,

$$
G_{P}=\left(I, E_{\gamma}\right)
$$

where $I$ is the set of intersections present in the entire urban area, the data for traffic flow (like traffic density, travel time etc.) and geometric design of roads is associated to the edges, while the data about the type of intersection is defined as the property of the nodes. The set of edges

$$
E_{\gamma}=\{(x, y) \mid x \gamma y\}, \gamma \in \Gamma, \forall x, y \in I, x \neq y
$$

exists between two adjacent intersections where $\Gamma=\{D\}$.

For dual graph,

$$
G_{D}=\left(R, E_{\psi}\right)
$$

where $R$ is the set of all the road segments, the spatial relations between two road segments are labeled on the edges connecting them. The set of edges

$$
E_{\psi}=\{(x, y) \mid x \psi y\}, \psi \in \Psi, \forall x, y \in R, x \neq y
$$

exists between two intersecting road segments. The set of type of relations between these road segments is $\Psi=\{A R, R O\}$ where, $A R$ represents accessibility relation, which describes if a road segment is directly accessible from another road segment on the basis of the values in adjacency matrix for the road network [27], and $R O$ explains the relative orientation between two road segments in a local reference frame. Combination of graphs defined in this section will provide a much broader picture about the urban traffic.

\section{GRANULARITY}

In this section, we will explain that how different viewpoints discussed above define different levels of detail, at which a road network can be envisaged.

Granularity of a model defines an analogy which compares the levels of abstraction in the model to granules, where finer granules are equivalent to higher levels of detail and coarser granules to lower levels [28]. These levels allow to segregate the information available from the entire model and process only the pertinent information, and thus make the model more robust and efficient.

Considering the entire urban area for which we want to understand and visualize the road traffic, the graph $G$ defined in the previous section gives us the information at the finest level of granularity. It considers all the objects present on all the road segments in that area. However, when we focus on a single road segment, two types of granularity emerge: Carriageway based granularity and Sector based granularity (similar to granular computing in [29]).

\section{A. Carriageway based granularity}

When the road segment is divided into two carriageways, the vehicles are categorized into two groups, based on the 
relation they have with the carriageways. These groups define the entities and corresponding spatial relations define the edges of the graph $G_{L i}$ for $i$ th road segment (Fig. 4).

A road segment $R_{i}$ divided into two carriageways $l 1$ and $l 2$ having three vehicles moving in one direction and two moving in the opposite direction is shown in Fig. 4a. The physical objects included in this example are buildings $(B 1, B 2)$, vertical structures (sign boards $(S 1, S 2)$ ), left and right roadside $(F r, F l)$, road marking (center line $C L)$ and pedestrians $(P 1$, $P 2)$. Fig. 4b shows corresponding graphs for two different levels of abstraction. At the finer level of detail, the graph (part of $G_{i}$ ) represents the relations between each vehicle and objects/pedestrians in its vicinity. It is noteworthy that if the view between a vehicle and an object is obstructed, then the relation between those two entities is not included. This is assumed to be a limitation of the sensors. At the coarser level, the graph $G_{L i}$ is defined, the nodes of which represent the traffic for both carriageways. Fig. 4c shows a graph similar to the one for the finer level in Fig. 4b, but with a single node for each entity. This graph structure represents the storage and implementation view of the graph in Fig. 4b, which shows the conceptual view for better understanding of the relations.

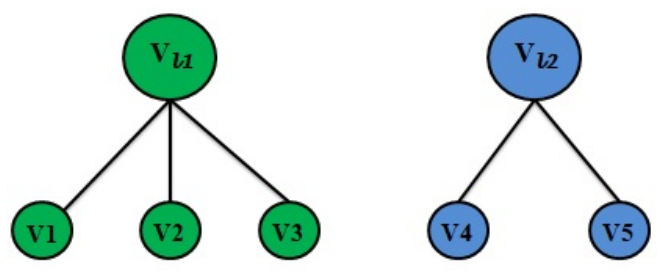

Fig. 5. Bipartite graph $G_{T}$ showing the categorization of vehicles into two groups represented using different colors

To explain the transition from $G_{i}$ to $G_{L i}$, we define a relation $\delta \in T \backslash\{D C, E Q, E C, P O, T P P i, N T P P i\}$ (topological relations in accordance with RCC8) between each vehicle $v \in V_{i}, V_{i} \subseteq V$ and carriageway $l k \in L, k=1,2$ describing if $v$ is a tangential proper part $(T P P)$ or nontangential proper part $(N T P P)$ of $l k$. According to value of $\delta$, we can categorize the vehicles into two groups. This categorization is shown in Fig. 5. It represents a bipartite graph $G_{T}=\left(Y_{i}, V_{i}, \sigma_{i}\right)$, in which one set of nodes is the set of vehicles $V_{i}$ and the other is set of groups of vehicles $Y_{i}$ for $i$ th road segment. The set of relations in this graph is

$$
\begin{gathered}
\sigma_{i}=\{(x, y) \mid y \text { belongsTo } x\} \\
\forall x, y\left(\left(x=V_{l k} \in Y_{i}\right) \wedge\left(y \in V_{i} \mid y \delta l k, l k \in L\right), k=1,2\right)
\end{gathered}
$$

The purpose to define a different graph $G_{T}$ is to make the transition from $G_{i}$ to $G_{L i}$ more clear.

\section{B. Sector based granularity}

When we consider that a road segment is divided into non-overlapping sectors, another type of granularity comes into play. At a finer level, we divide the road segment into set of sectors containing some number of vehicles. For each sector, we consider the relations between the vehicles and the objects/pedestrians present in that sector, and do not consider inter-sector relations.

In Fig. 6a, two arbitrary levels of sector based granularity are shown. At the finer one, a road segment is divided into two sectors. As a result, the entities present on/around that road segment are also divided. At a coarser level, the data related to both of them is considered. Fig. 6b shows the two distinct graphs for sectors $A_{1}^{j}$ and $A_{2}^{j}$. When these sectors are combined at a coarser level of abstraction, a single graph represents the relations for sector $A_{1}^{j+1}$, as shown in Fig. 6c. It is noteworthy that if, at a level of detail, an entity is shared by two consecutive sectors, then its corresponding relations are ignored at that level, but are considered at a higher level. For example, the relations which include vehicle $V 2$ or signboard $S 1$ are ignored in both the graphs in Fig. 6b. But at a coarser level of detail, when the two sectors are combined, the relations between all the entities included in the coarser sector are added into the graph. The transition from one level to another level of abstraction in this type of granularity depends on the operator defined, which may change the way the sectors are combined. This operator will take as inputs the graphs for finer sectors, and combine them to form a graph for a coarser sector. The definition of this operator is left for future.

\section{CONCLUSiOnS AND Future WORK}

In this paper, we present preliminary ideas to visualize urban road traffic, while taking into consideration different granularity and structure of the road network, which serve as a base for the development of a qualitative graphical model. This model is intended to improve the perception of the environment around a vehicle while describing the behavior of different traffic participants, as well as understanding the flow of traffic in a given urban area. We also define different sets of entities and relations for different levels of abstraction.

We are working on defining the mathematical operator (or function) to shift between different levels of abstraction in sector based granularity. When considering multiple road segments, the traffic data at intersections needs to be included and a dynamic intersection-centric graph, different from primal graph $G_{P}$, needs to be defined, which when combined with road segment-centric graph $G_{i}$, will help to describe the urban traffic flow. We intend to include temporal, in addition to spatial, relations between the entities, which will make the model dynamic. An ontology of entities present in a general model will be defined so that the number of relations between any two entities in the model can be reduced. We will implement the concepts presented in this paper using the data acquired from mapping services (like OpenStreetMaps and Google Maps), internal and external sensors, and the traffic data collected by CEREMA for the city of Rouen, in future.

\section{ACKNOWLEDGMENTS}

This work takes part in the DAISI project. This project has been funded with the support from the European Union with the European Regional Development Fund (ERDF) and from the Regional Council of Normandy. 


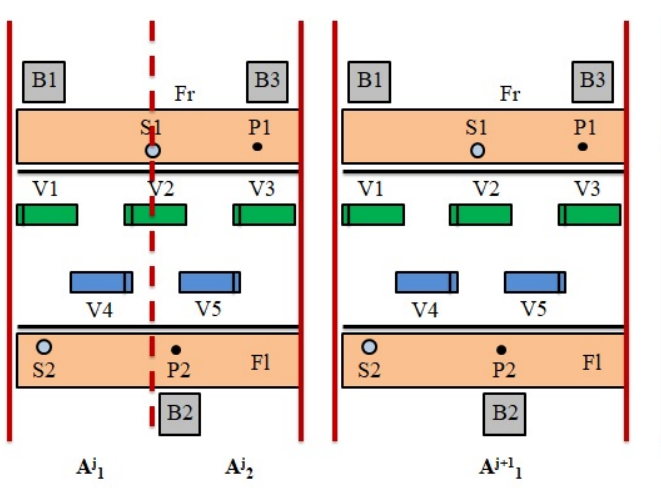

(a)

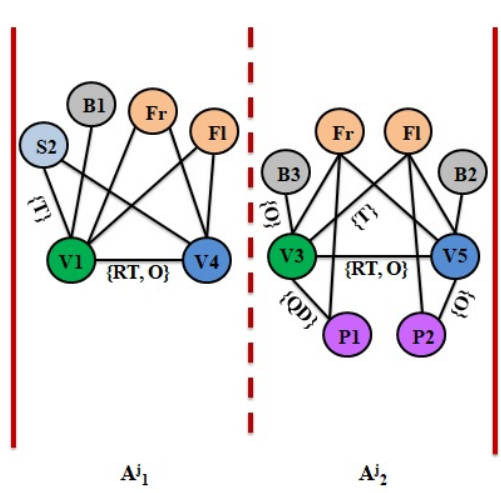

(b)

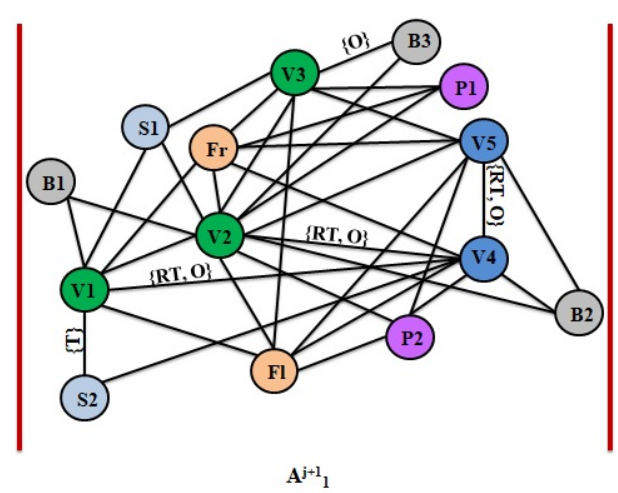

(c)

Fig. 6. (a) Two arbitrary levels of sector based granularity. At a finer level $j$, road segment is divided into two sectors $A_{1}^{j}$ and $A_{2}^{j}$, and at next coarser level $j+1$ these sectors combine to form single sector $A_{1}^{j+1}$ (b) Corresponding graphs for $A_{1}^{j}$ and $A_{2}^{j}$ (c) Corresponding graph for $A_{1}^{j+1}$. All the relations are not shown to make the image more readable

\section{REFERENCES}

[1] K. Nagel and M. Schreckenberg, "A cellular automaton model for freeway traffic," Journal de physique I, vol. 2, no. 12, pp. 2221-2229, 1992.

[2] I. Prigogine and R. Herman, Kinetic theory of vehicular traffic. Elsevier, New York, 1971.

[3] J. Holtzman, J. Hui, N. Moayeri, I. Seskar, H. Varma, J. Yip, S. Maric, and T. Williams, "A vehicular traffic GIS and simulator for route guidance on NY/NJ highways," in Proc. IEEE Vehicle Navigation and Information Systems Conference, Oct 1993, pp. 367-372.

[4] C. Claramunt, E. Peytchev, and A. Bargiela, "A real-time GIS for the analysis of a traffic system," in IEEE Africon, vol. 1, 1999, pp. 15-20.

[5] J. Levinson and S. Thrun, "Robust vehicle localization in urban environments using probabilistic maps," in International Conference on Robotics and Automation (ICRA), 2010, pp. 4372-4378.

[6] P. Stone, R. Brooks, E. Brynjolfsson, R. Calo, O. Etzioni, G. Hager, J. Hirschberg, S. Kalyanakrishnan, E. Kamar, S. Kraus, K. LeytonBrown, D. Parkes, W. Press, A. Saxenian, J. Shah, M. Tambe, and A. Teller, "Artificial intelligence and life in 2030. One hundred year study on artificial intelligence: Report of the 2015-2016 study panel," Stanford University, Stanford, CA, Tech. Rep., September 2016. [Online]. Available: http://ai100.stanford.edu/2016-report

[7] R. Parker and S. Valaee, "Vehicle localization in vehicular networks," in IEEE Vehicular Technology Conference, Sept 2006, pp. 1-5.

[8] S. Annell, A. Gratner, and L. Svensson, "Probabilistic collision estimation system for autonomous vehicles," in 2016 IEEE 19th International Conference on Intelligent Transportation Systems (ITSC), Nov 2016, pp. $473-478$.

[9] W. Guan, "Modeling traffic flow characteristics with qualitative and quantitative knowledge," in Proc. IEEE International Conference on Intelligent Transportation Systems, vol. 1, Oct 2003, pp. 151-155.

[10] Y. Li and D. Sun, "Microscopic car-following model for the traffic flow: the state of the art," Journal of Control Theory and Applications, vol. 10, no. 2, pp. 133-143, 2012.

[11] G. Del Mondo, "A Spatio-Temporal Graph-based Model for the Evolution of Geographical Entities," Theses, Université de Bretagne occidentale - Brest, Oct 2011.

[12] P. P.-S. Chen, "The entity-relationship model-toward a unified view of data," ACM Transactions on Database Systems, vol. 1, no. 1, pp. 9-36, 1976.

[13] K. Hornsby and M. J. Egenhofer, "Qualitative representation of change," in International Conference on Spatial Information Theory, 1997, pp. $15-33$.

[14] M. J. Egenhofer, "A formal definition of binary topological relationships," in International Conference on Foundations of Data Organization and Algorithms. Springer, 1989, pp. 457-472.

[15] C. Freksa, "Using orientation information for qualitative spatial reasoning," in Theories and methods of spatio-temporal reasoning in geographic space, ser. Lecture Notes in Computer Science. Springer, Berlin, 1992, vol. 639, pp. 162-178.

[16] D. Hernández, "Maintaining qualitative spatial knowledge," in European Conference on Spatial Information Theory. Springer, 1993, pp. 36-53.

[17] A. U. Frank, "Qualitative spatial reasoning: Cardinal directions as an example," International Journal of Geographical Information Science, vol. 10, no. 3, pp. 269-290, 1996

[18] E. Clementini, P. Di Felice, and D. Hernández, "Qualitative representation of positional information," Artificial intelligence, vol. 95, no. 2, pp. 317-356, 1997.

[19] J. Renz, "A spatial odyssey of the interval algebra: 1. directed intervals," in Proceedings of the 17th International Joint Conference on Artificial Intelligence - Volume 1, ser. IJCAI'01. Morgan Kaufmann Publishers Inc., 2001, pp. 51-56.

[20] S. Porta, P. Crucitti, and V. Latora, "The network analysis of urban streets: a primal approach," Environment and Planning B: planning and design, vol. 33, no. 5, pp. 705-725, 2006.

[21] _ _ "The network analysis of urban streets: a dual approach," Physica A: Statistical Mechanics and its Applications, vol. 369, no. 2, pp. 853866, 2006

[22] Q.-J. Kong, Q. Zhao, C. Wei, and Y. Liu, "Efficient traffic state estimation for large-scale urban road networks," IEEE Transactions on Intelligent Transportation Systems, vol. 14, no. 1, pp. 398-407, 2013.

[23] S. Kamran and O. Haas, "A multilevel traffic incidents detection approach: Identifying traffic patterns and vehicle behaviours using realtime GPS data," in Intelligent Vehicles Symposium, 2007 IEEE. IEEE, 2007, pp. 912-917.

[24] D. A. Randell, Z. Cui, and A. G. Cohn, "A spatial logic based on regions and connection." in Proceedings of the Third International Conference on Principles of Knowledge Representation and Reasoning, vol. 92, 1992, pp. 165-176.

[25] M. Sridhar, A. G. Cohn, and D. C. Hogg, "Benchmarking qualitative spatial calculi for video activity analysis," in Proceedings IJCAI Workshop Benchmarks and Applications of Spatial Reasoning. Leeds, 2011, pp. $15-20$

[26] R. Röhrig, "A theory for qualitative spatial reasoning based on order relations," in Proceedings of the Twelfth National Conference on Artificial Intelligence (Vol. 2), ser. AAAI'94. American Association for Artificial Intelligence, 1994, pp. 1418-1423.

[27] T. Cheng, J. Haworth, and J. Wang, "Spatio-temporal autocorrelation of road network data," Journal of Geographical Systems, vol. 14, no. 4, pp. 389-413, 2012.

[28] C. M. Keet, "A taxonomy of types of granularity," in IEEE International Conference on Granular Computing, May 2006, pp. 106-111.

[29] B. Płaczek, "The granular computing implementation for road traffic video-detector sampling rate finding," Transport Problems, vol. 4, pp. 55-62, 2009. 